\title{
Lattice study on exotic vector charmonium relevant to $X(4260)$
}

Ying Chen*; Wei-Feng Chiu, Long-Cheng Gui, Jian Liang, Zhaofeng Liu, and Yi-Bo Yang

Institute of High Energy Physics and Theoretical Physics Center for Science Facilities,

Chinese Academy of Sciences, Beijing 100049, China

E-mail: chenydihep.ac.cn, wfchiudihep.ac.cn, guilongchengdihep.ac.cn,

liangiiandihep.ac.cn, liuzfdihep.ac.ad ybyangdihep.ac.cn

\section{Keh-Fei Liu}

Department of Physics and Astronomy, University of Kentucky, Lexington, KY 40506, USA

E-mail: Liuepa.uky.edu

In the quenched approximation and with very high statistics, a heavy vector charmonium state, with a mass of $4.33(2) \mathrm{GeV}$, is disentangled from the conventional charmonium states by using spatially extended hybrid-like interpolating field operators. Through a simultaneous fit of correlation functions built from different operators, the leptonic decay constant this state is estimated to be $31(15) \mathrm{MeV}$, which gives a much smaller leptonic decay width of $23(20) \mathrm{eV}$ in comparison with that of conventional vector charmonia. The connection of this state with $X(4260)$ is also discussed.

31st International Symposium on Lattice Field Theory - LATTICE 2013

July 29 - August 3, 2013

Mainz, Germany

* Speaker.

$\dagger$ This work is supported in part by the National Science Foundation of China (NSFC) under Grants No.10835002, No.11075167, No.11105153 and also by the U.S. DOE Grants No. DE-FG05-84ER40154. Y. C. and Z. L. also acknowledge the support of NSFC and DFG (CRC110). 


\section{Introduction}

$X(4260)$ was first observed by BABAR and confirmed by Belle and CLEO in the initial state radiation (ISR) process $e^{+} e^{-} \rightarrow \gamma_{\mathrm{ISR}} X \rightarrow \gamma_{\mathrm{ISR}} J / \psi \pi \pi$ [四, [, 四] with the resonance parameter $M_{X}=$ $4259 \pm 8_{-6}^{+2} \mathrm{MeV}$ and $\Gamma_{X}=88 \pm 23_{-4}^{+6} \mathrm{MeV} . X(4260)$ has definitely the quantum number $J^{P C}=1^{--}$ and its leptonic decay width is estimated as $\Gamma\left(X \rightarrow e^{+} e^{-}\right) B r(X \rightarrow J / \psi \pi \pi)=5.5 \pm 1.0_{-0.7}^{+0.8} \mathrm{eV}$ [四], which is in contrast to the similar combined width $672 \pm 45 \mathrm{eV}$ of $\psi(3686)$. As such, $X(4260)$ must have an unusual small $e^{+} e^{-}$width or a very small coupling to $J / \psi \pi \pi$. The nature of $X(4260)$ is now a hot topic and has been tentatively assigned to be a tetraquark state, a molecular state, or a hybrid charmonium [四, [1, 目] by phenomenological studies.

As far as a hybrid charmonium is concerned, it is usually conjectured as a bound state made up of a charm quark-antiquark pair and a (constituent) gluon in the picture of the constituent quark model. In this work, we carry out a lattice study on the possible vector hybrid charmonium (denoted as $\psi_{\text {hyb }}\left(J^{P C}=1^{--}\right)$in this work) in the quenched approximation. Since it has the same quantum number as the conventional vector charmonium such as $J / \psi, \psi^{\prime}$, etc., it is a challenging task to disentangle $\psi_{\text {hyb }}$ from them. For this purpose, we split the commonly used hybrid operator $\bar{c} \Gamma c F$ ( $F$ refers to the gluon field strength tensor) with the quark bilinear separating from $F$ with a spatial distance. This kind of operators is expected to reflect the exotic picture of a hybrid-like state that the constituent $c \bar{c}$ pair gets a center-of-mass motion by recoiling against the additional gluonic degree of freedom, such that they may couple preferably to $\psi_{\text {hyb }}$ while their coupling to the conventional vectors may be suppressed. If $\psi_{\text {hyb }}$ can be reliably singled out, its leptonic decay constant can be subsequently derived through its coupling to the vector current. We will also discuss the connection of $\psi_{\text {hyb }}$ with X(4260).

\section{Numerical details}

For the vector charmonium $\left(J^{P C}=1^{--}\right)$of a possible hybrid configuration $c \bar{c} g$, the commonly used simple and straightforward local operator is $O_{i}^{(H)}(x)=\bar{c}^{a}(x) \gamma_{5} c^{b}(x) B_{i}^{a b}(x)$, where $a, b$ are color indices, $i$ the spatial index, and $B_{i}^{a b}(x)=\frac{1}{2} \varepsilon_{i j k} F_{j k}^{a b}$ the chromomagnetic field tensor. On the other hand, the conventional quark bilinear operator for the vector is $O_{i}^{(M)}=\bar{c} \gamma_{i} c(x)$. Through the FoldyWouthuysen-Tani transformation [ [] ], these operators can be decomposed into the non-relativistic expressions in terms of the Pauli spinors $\phi / \phi^{\dagger}$ which annihilates/creates a charm quark, and $\chi / \chi^{\dagger}$ which creates/annihilates a charm antiquark. Thus to the lowest order of the nonrelativistic approximation, the operators $O_{i}^{(H)}(x)$ and $O_{i}^{(M)}$ are reduced to be

$$
O_{i}^{(H)} \equiv \bar{c}^{a} \gamma_{5} c^{b} B_{i}^{a b} \rightarrow \chi^{a \dagger} \phi^{b} B_{i}^{a b}+O\left(\frac{1}{m_{c}}\right), \quad O_{i}^{(M)} \equiv \bar{c}^{a} \gamma_{i} c^{a} \rightarrow \chi^{a \dagger} \sigma_{i} \phi^{a}+O\left(\frac{1}{m_{c}}\right) .
$$

Obviously the block $\chi^{a^{\dagger}} \phi^{b}$ of the $O_{i}^{(H)}$ operator is a spin singlet and color octet, while that of $O_{i}^{(M)}$ is a spin triplet and color singlet. If we go further to split spatially the operator $O_{i}^{(H)}$ into two parts, say, separate the quark bilinear $\bar{c}^{a} \gamma_{5} c^{b}$ from the chromomagnetic field tensor $B_{i}^{a b}$ with an explicit spatial displacement $\mathbf{r}$, in a fixed gauge (the Coulomb gauge in this work), we get a set of spatially extended operators,

$$
O_{i}^{(H)}(\mathbf{x}, t ; \mathbf{r})=\left(\bar{c}^{a} \gamma_{5} c^{b}\right)(\mathbf{x}, t) B_{i}^{a b}(\mathbf{x}+\mathbf{r}, t)
$$


In the nonrelativistic limit, there is a two-fold suppression for $O_{i}^{(H)}$ to couple to the conventional vector charmonia $\psi(n S)$ : First, the charm quark-antiquark pair is in the spin triplet in $\psi(n S)$ and the spin flipping is suppressed by the heavy quark mass; secondly, there is no center-of-mass motion of $c \bar{c}$ for $\psi(n S)$. Therefore, it is expected that the possible hybrid vector charmonium $\psi_{\text {hyb }}$ can be tackled more reliably from the correlation functions of this kind of operators.

The calculation of this work is performed in the quenched approximation on anisotropic lattices with the temporal lattice spacing much finer than the spatial one, say, $\xi=a_{s} / a_{t}=5$, where $a_{s}$ and $a_{t}$ are the spatial and temporal lattice spacing, respectively. Two lattices are used and 1000 configurations for each lattice system are generated with the tadpole improved gauge ac-

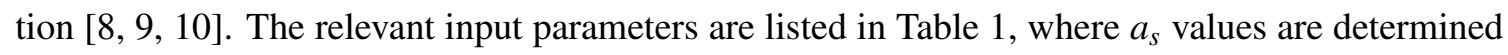
from $r_{0}^{-1}=410(20) \mathrm{MeV}$. The spatial extension of both lattice is $\sim 1.7 \mathrm{fm}$, which is large enough for charmonia. We use the tadpole-improved clover action [ए]] for the charm quark. The parameters in the actions are tuned carefully by requiring that the physical dispersion relations of vector and pseudoscalar mesons are correctly reproduced at each bare quark mass [प]]. The bare charm quark mass is set by the physical mass of $J / \psi, m_{J / \psi}=3.097 \mathrm{GeV}$. The finite $a_{s}$ effects are found to be small in this kind of lattice setup by testing the spectrum of $1 S$ and $1 P$ charmonia [[13]].

\begin{tabular}{cccccc}
\hline \hline$\beta$ & $\xi$ & $a_{s}(\mathrm{fm})$ & $L a_{s}(\mathrm{fm})$ & $L^{3} \times T$ & $N_{\text {conf }}$ \\
\hline 2.4 & 5 & $0.222(2)$ & 1.78 & $8^{3} \times 96$ & 1000 \\
2.8 & 5 & $0.138(1)$ & 1.66 & $12^{3} \times 144$ & 1000 \\
\hline \hline
\end{tabular}

Table 1: The input parameters for the calculation. Values for the coupling $\beta$, anisotropy $\xi$, the lattice spacing $a_{s}$, lattice size, and the number of measurements are listed.

In order to enhance the signal of the possible $\psi_{\text {hyb }}$, we use a source operator $O_{i}^{(W)}(\tau)=$ $\sum_{\mathbf{y}, \mathbf{z}} \bar{c}^{a}(\mathbf{y}, \tau) \gamma_{5} B_{i}^{a b}(\mathbf{z}, \tau) c^{b}(\mathbf{z}, \tau)$ to calculate the two point function,

$$
\begin{aligned}
C^{(H)}(r, t) & =\frac{1}{3 T N_{r}} \sum_{\tau=0}^{N_{t}-1} \sum_{|\mathbf{r}|=r} \sum_{\mathbf{x}, i}\left\langle 0\left|O_{i}^{(H)}(\mathbf{x}, t+\tau ; \mathbf{r}) O_{i}^{(W) \dagger}(\tau)\right| 0\right\rangle \\
& =\frac{1}{3 T N_{r}} \sum_{\tau=0}^{N_{t}-1} \sum_{|\mathbf{r}|=r \mathbf{x}, \mathbf{y}, \mathbf{z}, i} \sum_{i}\left\langle S_{F}^{\dagger}(\mathbf{x}, t+\tau ; \mathbf{z}, \tau) B_{i}(\mathbf{x}+\mathbf{r}, t+\tau) S_{F}(\mathbf{x}, t+\tau ; \mathbf{y}, \tau) B_{i}^{\dagger}(\mathbf{z}, \tau)\right\rangle
\end{aligned}
$$

where $\tau$ refers to the source time slice and $N_{r}$ is the degeneracy of the displacement $\mathbf{r}$ with the same $r$. In practice, the gauge configurations are fixed to the Coulomb gauge first. In order to increase the statistics additionally, for each configuration we calculate $T$ charm quark propagators $S_{F}(\vec{x}, t ; \tau)$ by setting a wall source on each time slice $\tau$, which permits us to average over the temporal direction when calculating the two-point functions.

In the data analysis, the correlation functions $C^{(H)}(\mathbf{r}, t)$ with different $r$ are simultaneously fitted using the function form

$$
C^{(H)}(r, t) \equiv \sum_{n=1} \frac{1}{2 m_{n}} Z_{n}^{(H)}(r) Z_{n}^{(W) *} e^{-m_{n} t}
$$



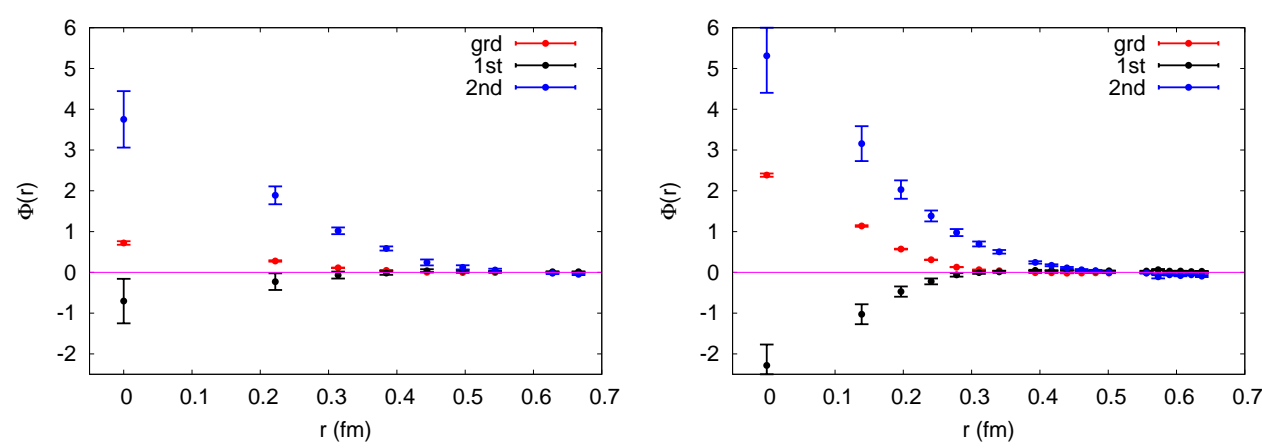

Figure 1: Plots of $\Phi_{n}(r)=\frac{1}{2 m_{n}} Z_{n}^{(H)}(r) Z_{n}^{(W) *}$ with respect to $r$ (in physical units) for three three lowest states ( the left panel is for the $\beta=2.4$ case, and the right panel for $\beta=2.8$ ), whose masses are fitted to be 3.091(9) GeV, 3.85(25) GeV and 4.51(13) GeV for $\beta=2.4$, and 3.084(2) GeV, 3.776(72) GeV, and 4.43(10) $\mathrm{GeV}$ for $\beta=2.8$.

through the correlated minimal- $\chi^{2}$ fit method with the jackknife covariance matrix. Here the parameter $Z_{n}^{(X)}$ is defined as

$$
\left\langle 0\left|O_{i}^{(X)}\right| V_{n}(\mathbf{p}, r)\right\rangle=Z_{n}^{(X)} \varepsilon_{i}(\mathbf{p}, r), \quad X=H, W .
$$

Figure $\mathbb{U}$ shows the plots of $\Phi_{n}(r)=\frac{1}{2 m_{n}} Z_{n}^{(H)}(r) Z_{n}^{(W) *}$ with respect to $r$ (in physical units) for the three lowest states (the left panel is for $\beta=2.4$, and the right panel for $\beta=2.8$ ), whose masses are fitted to be 3.091(9) GeV, 3.85(25) GeV and 4.51(13) GeV for $\beta=2.4$, and 3.084(2) GeV, 3.776(72) GeV, and 4.43(10) $\mathrm{GeV}$ for $\beta=2.8$. From the figure one sees that $\Phi_{1}(r)$ and $\Phi_{2}(r)$ damp more rapidly and are close to zero at $r \sim 0.3 \mathrm{fm}$ where $\Phi_{3}(r)$ is still relatively large. The fitted masses and $\Phi_{n}$ signal that the two lowest states correspond to $J / \psi$ and $\psi(3686)$ and the third state might be a special state of different internal structure from conventional vector charmonia. In order to test the reliability of the fitting strategy mentioned above, we also carry out a similar analysis to the correlation functions $C^{(M)}(r, t)$ of the spatially extended version of operator $O^{(M)}$ on the $\beta=2.4$ lattice. In the fitting procedure, we fix a maximal $t$ (denoted by $t_{\max }$ ) and vary the lower bound $t_{\min }$ of the fit window. We find the masses of the three lowest states keep constant to some extent for a series of $t_{\min }$, as shown in the right panel of Fig. ㅁ. We average the masses in this range with each value weighted by its error and get the values $m_{1}=3.097(1) \mathrm{GeV}, m_{2}=3.679(19) \mathrm{GeV}$, and $m_{3}=4.007(57) \mathrm{GeV}$, respectively. These three states may correspond to $J / \psi, \psi(3686)$, and $\psi(4040)$. We also plot the $\Phi_{n}(r / a)$ 's (normalized as $\left.\Phi_{n}(0)=1\right)$ in the left panel of Fig. Z, where one can find the radial node structure expected by the non-relativistic potential model.

Motivated by this observation, in order for the state to be identified more clearly, we combine linearly the correlation functions $C^{(H)}(r, t)$ at two specific $r_{1}$ and $r_{2}$ as

$$
C(\omega, t)=C^{(H)}\left(r_{1}, t\right)+\omega C^{(H)}\left(r_{2}, t\right)
$$

with $\omega$ a tunable parameter. The separations $r_{1}$ and $r_{2}$ are chosen with the requirement that the signal-to-noise ratio is good enough and spectral weights of the corresponding correlation functions are very different for the would-be conventional charmonia and the hybrid-like state. As such we 

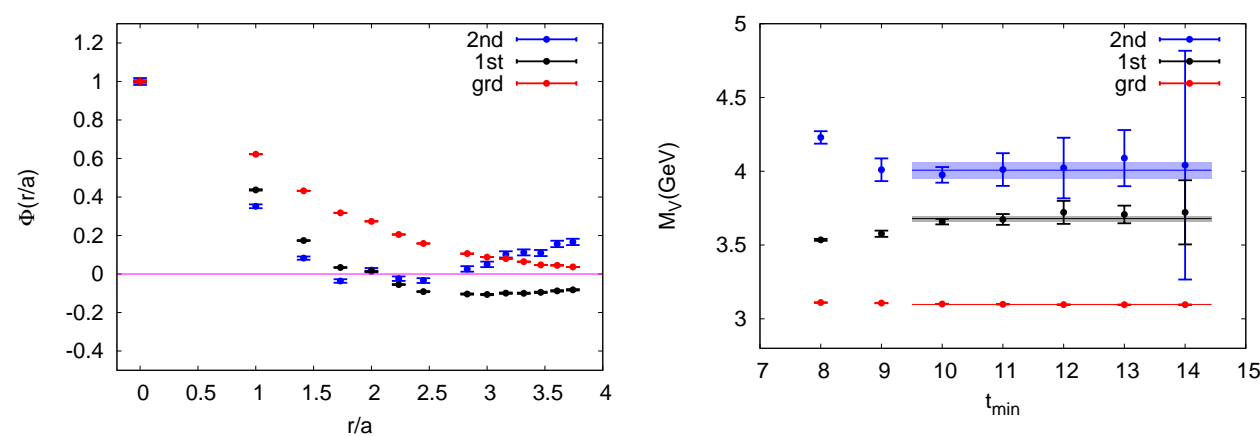

Figure 2: Left panel: $\Phi_{n}(r / a)$ 's (normalized as $\Phi_{n}(0)=1$ ) of the three lowest states from fits to $C^{(M)}(r, t)$ of the spatially extended version of operator $O^{(M)}$ on the $\beta=2.4$ lattice. Right panel: Masses of the three lowest states as functions of $t_{\min }$.

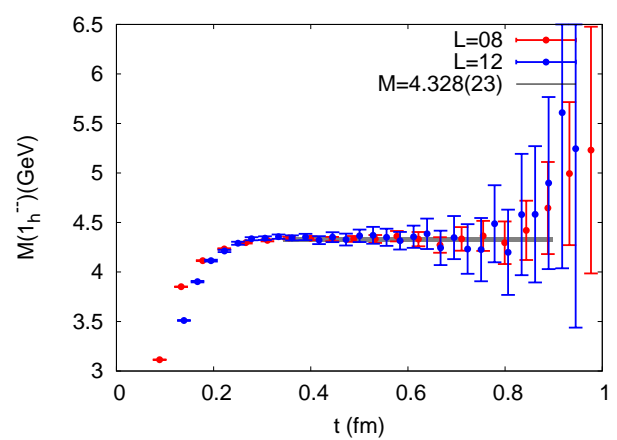

Figure 3: The effective mass plateaus of $C(\omega, t)$ 's for $\beta=2.4$ and $\beta=2.8$.

tune the parameter $\omega$ for $C(\omega, t)$ to have an effective mass plateau as long as possible. In Fig. [] we plot the effective mass plateaus of $C(\omega, t)$ 's for $\beta=2.4$ and $\beta=2.8$ with respectively optimally tuned $\omega$. The axes are labeled with the physical units. One can see that both the plateaus are laid on each other at a mass of roughly $4.33 \mathrm{GeV}$. It is clearly seen in the above study that the hybrid-like operator $O_{i}^{(H)}(\mathbf{x}, t ; \mathbf{r})$ couples surely preferable to the state with a mass of roughly $4.33(2) \mathrm{GeV}$, which confirms our speculation based on the non-relativistic picture. With this observation, it is highly conjecturable that this state is a hybrid-like charmonium.

Since this hybrid-like charmonium can be disentangled from the conventional charmonium with the prescription above, its leptonic decay constant can be investigated accordingly. It is known that the leptonic decay constant $f_{V}$ of a vector meson state $V$ is defined by

$$
\left\langle 0\left|J_{\mu}^{(\mathrm{em})}(0)\right| V(\vec{p}, r)\right\rangle=m_{V} f_{V} \varepsilon_{\mu}(\vec{p}, r),
$$

where $J_{\mu}^{(\mathrm{em})}(0)$ is the electromagnetic current and $\varepsilon_{\mu}(\vec{p}, r)$ is the polarization vector of $V$ at momentum $\vec{p}$. For vector charmonium states, $J_{\mu}^{(\mathrm{em})}(0)$ can be approximated by $\bar{c} \gamma_{\mu} c(0)$ according to the Okubo-Zweig-Iizuka rule. On the lattice with a finite lattice spacing, the vector current $\bar{c} \gamma_{\mu} c(0)$ in the continuum theory is not conserved any more and should be renormalized properly. We adop$\mathrm{t}$ the nonperturbative renormalization strategy proposed by Ref. [U4]] and get the renormalization 


\begin{tabular}{lcccc}
\hline \hline$\beta$ & $M(J / \psi)(\mathrm{GeV})$ & $f_{J / \psi}(\mathrm{MeV})$ & $M\left(\psi_{\mathrm{hyb}}\right)(\mathrm{GeV})$ & $f_{\psi_{\mathrm{hyb}}}(\mathrm{MeV})$ \\
\hline 2.4 & $3.076(4)$ & $428(7)$ & $4.43(7)$ & $32(20)$ \\
2.8 & $3.082(1)$ & $378(6)$ & $4.40(7)$ & $31(11)$ \\
\hline Exp. & 3.097 & $407(5)$ & $\cdots$ & $\cdots$ \\
\hline \hline
\end{tabular}

Table 2: Preliminary results of masses and decay constants of $J / \psi$ and $\psi_{\text {hyb }}$.

constants $Z_{V}^{(s)}=1.39(2)$ for $\beta=2.4$ and $Z_{V}^{(s)}=1.11(1)$ for $\beta=2.8$ [एँ3], ㅁ5], where the superscrip$\mathrm{t} s$ stands for the spatial components of the vector current. Note that the spatial components of $J_{\mu}^{(\mathrm{em})}(x)$ is exact the normal quark bilinear operator $O_{i}^{(M)}$ for vector mesons, so the matrix elements in Eq. (2.7]) can be derived from the corresponding correlation functions when the operator $O_{i}^{(M)}$ is involved. Along with the vector current renormalization constant $Z_{V}^{(s)}$, one can derive the decay constant $f_{V}$ for a vector charmonium $V$ if it can be singled out unambiguously.

In addition to $C^{(H)}(\mathbf{r}, t)$, we also calculate other two categories of correlation functions,

$$
\begin{aligned}
& C^{(J)}(t)=\frac{1}{3} \sum_{\mathbf{x}, i}\left\langle 0\left|J_{i}(\mathbf{x}, t) O_{i}^{(W) \dagger}(0)\right| 0\right\rangle \equiv \sum_{n} \frac{1}{2 m_{n}} Z_{n}^{(J)} Z_{n}^{(W) *} e^{-m_{n} t}, \\
& C^{(W)}(t)=\frac{1}{3} \sum_{i}\left\langle 0\left|O_{i}^{(W)}(t) O_{i}^{(W) \dagger}(0)\right| 0\right\rangle \equiv \sum_{n} \frac{1}{2 m_{n} V_{3}} Z_{n}^{(W)} Z_{n}^{(W) *} e^{-m_{n} t},
\end{aligned}
$$

where $m_{n}$ is the mass of the $n$-th state and the parameter $Z_{n}^{(X)}$ with $X$ referring to $J$ or $W$.

Accordingly the leptonic decay constant $f_{V_{n}}$ can be derived from $Z_{n}^{(J)}$ from the definition Eq. (Z.Z) as

$$
f_{V_{n}}=Z_{V}^{(s)} Z_{n}^{(J)} / m_{n}
$$

In the data analysis stage, we carry out the simultaneous correlated minimal- $\chi^{2}$ fit to the correlation functions $C^{(J)}(t), C^{(H)}(r, t)$, and $C^{(W)}(t)$ using the function forms in Eqs. $([2.4,2.8)$ with parameters $m_{n}, Z_{n}^{(X)}$. The preliminary results of leptonic decay constants of $J / \psi$ and $\psi_{\text {hyb }}$ derived in this kind of fit are given in Tab. $\square$ in physical units. The decay constant of $J / \psi$ reproduces more or less the experimental result $f_{J / \psi}=407(5) \mathrm{MeV}$ on the two lattices. For the likely exotic vector charmonium $\psi_{\text {hyb }}$, we get its decay constant as

$$
f_{\psi_{\mathrm{hyb}}}=31(15) \mathrm{MeV}
$$

which is roughly one order of magnitude smaller than that of $J / \psi$, although the error is still very large. Thus the leptonic decay width of the $\psi_{\text {hyb }}$ state can be determined to be

$$
\Gamma\left(\psi_{\text {hyb }} \rightarrow e^{+} e^{-}\right)=\frac{16 \pi}{27} \alpha_{\mathrm{QED}}^{2} \frac{f_{\psi_{\text {hyb }}}^{2}}{M_{\psi_{\text {hyb }}}} \approx 23(20) \mathrm{eV},
$$

which is much smaller than that of conventional vector charmonia (we use $\alpha_{\mathrm{QED}}=1 / 134$ at the charm quark mass scale). If $X(4260)$ is tentatively assigned to the $\psi_{\text {hyb }}$ state investigated in this study, according to the combined partial width

$$
\Gamma\left(X \rightarrow e^{+} e^{-}\right) \operatorname{Br}(X \rightarrow J / \psi \pi \pi)=5.5 \pm 1.0_{-0.7}^{+0.8} \mathrm{eV}
$$


the branch ratio of $X(4260)$ decaying into $J / \psi \pi^{+} \pi^{-}$can be estimated to be

$$
\operatorname{Br}(X(4260) \rightarrow J / \psi \pi \pi) \sim 20(16) \%,
$$

which means that $J / \psi \pi \pi$ is one of the most important decay modes of $X(4260)$. This can naturally explain the fact that $X(4260)$ was only observed in this channel and does not show up the resonance structure in the R-value scan around $\sqrt{s}=4.26 \mathrm{GeV}$.

\section{Summary}

By using spatially extended operators that reflect the hybrid picture to some extent, a hybridlike vector charmonium $\psi_{\text {hyb }}$ with a mass of $4.33(2) \mathrm{GeV}$ is disentangled from the conventional vector charmonia. In addition, through a simultaneous multi-state fit to correlation functions built from the vector current operator and the hybrid operator mentioned above, a preliminary result of the leptonic decay constant of $\psi_{\text {hyb }}$ is extracted to be $f_{\psi_{\text {hyb }}}=31(15) \mathrm{MeV}$, which gives a very small leptonic decay width $\Gamma\left(\psi_{\text {hyb }} \rightarrow e^{+} e^{-}\right)=23(20) \mathrm{eV}$. The mass and the leptonic decay width of $\psi_{\text {hyb }}$ are compatible with the production and decay properties of $\mathrm{X}(4260)$.

\section{References}

[1] B. Aubert et al. (BABAR Collaboration), Phys. Rev. Lett. 95, 142001 (2005) [hep-ex/0506081].

[2] C.Z. Yuan et al. (Belle Collaboration), Phys. Rev. Lett. 99, 182004 (2007) [arXiv:0707.2541 (hep-ex)].

[3] T.E. Coan et al. (CLEO Collaboration), Phys. Rev. Lett. 96, 162003 (2006) [hep-ex/0602034].

[4] S.L. Zhu, Phys. Lett. B 625, 212 (2005) [hep-ph/0507025].

[5] F.E. Close and P.R. Page, Phys. Lett. B 628, 215 (2005) [hep-ph/0507199].

[6] E. Kou and O. Pene, Phys. Lett. B 631, 164 (2005) [hep-ph/0507119].

[7] L.L Foldy and S.A. Wouthuysen, Phys. Rev. 78, 29 (1950); S. Tani, Prog. Theor. Phys. 6, 267 (1951).

[8] C.J. Morningstar and M. Peardon, Phys. Rev. D 56, 4043 (1997) [hep-lat/9704011].

[9] C.J. Morningstar and M. Peardon, Phys. Rev. D 60, 034509 (1999) [hep-lat/9901004].

[10] Y. Chen et al., Phys. Rev. D 73, 014516 (2006) [hep-lat/0510074].

[11] C. Liu, J. Zhang, Y. Chen, J.P. Ma, Nucl. Phys. B 624, 360 (2002).

[12] S. Su, L. Liu, X. Li, and C. Liu, Int. J. Mod. Phys. A 21, 1015 (2006) [hep-lat/0412034], Chin. Phys. Lett. 22, 2198 (2005) [hep-lat/0505006].

[13] Y.-B. Yang et al., (CLQCD Collaboration), Phys. Rev. D 87, 014501 (2013) [arXiv:1206.2086 (hep-lat)].

[14] J.J. Dudek, R.G. Edwards, and D.G. Richards, Phys. Rev. D 73, 074507 (2006).

[15] L.-C. Gui et al. (CLQCD Collaboration), Phys. Rev. Lett. 110021601 (2013) [arXiv:1206.0125 (hep-lat)]. 